\title{
Abundances in a Large Sample of Stars in the LMC Disk II. Cu, Sc, and $s$-elements and Some Relationships Between Elements
}

\author{
L. Pompéia ${ }^{1}$, V. Hill ${ }^{2} \&$ M. Spite ${ }^{2}$ \\ ${ }^{1}$ Departamento de Astronomia, IAG - USP, Rua do Matão 1226, G307, Brazil \\ email: pompeia@astro.iag.usp.br \\ ${ }^{2}$ Paris-Meudon Observatory, 5 Place Jules Jansen, Meudon, France Camford, \\ email: Vanessa.Hill@obspm.fr, Monique.Spite@obspm.fr
}

\begin{abstract}
We have been using VLT - FLAMES facilities to obtain spectra of large samples of stars from the Large Magellanic Cloud in order to infer chemical abundances of some key elements. Target stars have been selected trying to sample as much as possible the hole metallicity range of the population. In the present paper we continue our report on chemical abundances for stars in the Inner Disk of the LMC. In our previous work (Pompéia, Hill \& Spite 2004) we have found a peculiar pattern for such stars, with a deficiency of $\alpha$ and Na relative to stars of the Galaxy. For the iron-peak elements we found an offset when compared to stars from the Galaxy which is difficult to explain when taking into account the present nucleosynthetic theories. In the present paper we report abundances derived from line synthesis for $\mathrm{Y}, \mathrm{Zr}, \mathrm{Ba}, \mathrm{Cu}$ and $\mathrm{Sc}$. We have found an interesting behavior for the $s$-process elements: while the heavy-s elements show supersolar values and overlap the galactic samples, the light- $s$ elements are underabundant with many subsolar values. $\mathrm{Cu}$ and Sc show deficient patterns compared to MW stars of similar metallicities.
\end{abstract}

Keywords. Galaxies: abundances, galaxies: evolution, galaxies: individual: LMC, stars: abundances,

\section{Introduction}

We have been witnessing a vast increase of data on the chemical abundances of stars in the Milky Way galaxy as, thanks to the operation of the large telescopes powered with potent spectrographs, of stars in galaxies other than our own. Two amazing results from this endeavor have been revealed. The first is that, for a given metallicity, stars from the Galaxy show very little dispersion in the chemical distributions of the different elements, and a smooth trend with metallicity is seen even in transition ranges between two different components (Venn et al. 2004, Gilmore \& Wyse 2004). The second is that stars from the Local Group and nearby galaxies show different chemical behavior compared to stars of the Galaxy with similar metallicities (Shetrone et al. 2003, Tolstoy et al. 2003, McWilliam \& Smecker-Hane 2004). Such results open a new field to the study of the galaxy formation processes, helping to understand how to connect chemical enrichment and galaxy evolution, and to shed light on questions as: how are the nucleosynthetic paths in systems with different SFH?

In our previous paper for the LMC Inner Disk sample (Pompéia, Hill \& Spite 2004, hereafter PHM04) we have found that these stars have peculiar abundances for many of the elements, showing deficient and for most of them, subsolar, abundance ratios relative to iron: $[\mathrm{X} / \mathrm{Fe}]$. The $\alpha$-elements $\mathrm{O}, \mathrm{Mg}, \mathrm{Ca}$ and $\mathrm{Si}$, as well as $\mathrm{Na}$, are underabundant when 
compared to the Galactic stars of the same metallicity. An amazing result is observed for the iron-peak group of elements. This group show a deficient pattern compared to galactic samples, with many subsolar values. The low $[\alpha / \mathrm{Fe}]$ abundance ratios may indicate a slower star formation history compared to that of the solar neighborhood.

In the present paper we report $\mathrm{Cu}, \mathrm{Sc}, \mathrm{Zr}, \mathrm{Y}$ and $\mathrm{Ba}$ abundances for this sample. We also discuss some relationships among these elements and those previously investigated (PHM04) such as the Na-Ni and Na-Mg trends. An overall paper will be published soon.

\section{Observations and Stellar Parameters}

We have used VLT Keuyen 8m telescope with GIRAFFE - FLAMES spectrograph to obtain high resolution spectra $(\mathrm{R}=18,500$ to 28,800$)$ of a sample of 67 LMC Inner Disk stars. MEDUSA configuration has been adopted with three different setups: H11, H13, and H14. Observations were made during the Science Verification of FLAMES on February 2003 and on March 2004. Reductions have been carried out using BLDRS (GIRAFFE Base Line Data Reduction Software) and MIDAS packages. Stellar parameters were first derived by using CTIO and 2MASS photometry. Temperatures were inferred from V-I and V-K colors, and we have adopted initial metallicities from the Ca infrared triplet calculated by Smecker-Hane et al. (in press). Spectroscopic stellar parameters have been inferred as follows: stellar effective temperatures have been calculated by the excitation equilibrium of Fe I lines; gravities and metallicities have been inferred by the ionization equilibrium of Fe I and Fe II lines; and microturbulent velocities have been inferred by demanding that Fe I lines of different equivalent widths give the same iron abundance.

\subsection{Abundance Determination and Results}

$\mathrm{Cu}, \mathrm{Sc}, \mathrm{Y}$ and $\mathrm{Ba}$ abundances have been derived from the spectrum synthesis of the $5782 \AA, 5657 \AA, 6127 \AA, 6435 \AA$ and $6496 \AA$ lines, respectively, and for Zr we have used two lines: $6127 \AA$ and $6134 \AA$. For Sc we have adopted HFS from Prochaska et al. (2000) and for Ba, from McWilliam (1998). The line fitting in the Solar Flux Atlas of Kurucz (Kurucz, Furenlid \& Brault 1984) give $[\mathrm{Cu} / \mathrm{Fe}]=+0.20 \mathrm{dex},[\mathrm{Sc} / \mathrm{Fe}]=0.0 \mathrm{dex},[\mathrm{Ba} / \mathrm{Fe}]=$ +0.20 dex, $[\mathrm{Zr} / \mathrm{Fe}]=-0.10 \operatorname{dex}($ mean for the two lines $) ;[\mathrm{Y} / \mathrm{Fe}]=0.00 \mathrm{dex}$. In the plots abundance ratios are not corrected for these values.

\subsection{1. $C u$ and $S c$}

In Figure 1 the $\mathrm{Cu}$ and $\mathrm{Sc}$ abundance distributions are shown. We have found an interesting pattern for $\mathrm{Cu}$ in the LMC stars. $\mathrm{Cu}$ is low $([\mathrm{Cu} / \mathrm{Fe}] \approx-0.60$ dex $)$ for metallicities lower than $[\mathrm{Fe} / \mathrm{H}]=-1.0$ dex. For higher metallicities there is a slow increasing trend with large scatter. Compared to the Galactic samples, we see that the different distributions overlap for very low metallicities, with galactic stars showing a steep increasing trend for $[\mathrm{Fe} / \mathrm{H}]>-1.4$ dex. In this metallicity range, LMC stars show underabundant ratios compared to galactic stars. Sc on the other hand shows supersolar ratios for the metal-poor tail, with a slow decreasing trend for increasing metallicities, and a flat behavior for $[\mathrm{Fe} / \mathrm{H}]>-1.20$ dex. Compared to stars of the Galaxy, [Sc/Fe] overlap the halo distribution, showing smaller ratios for higher metallicities.

Copper and scandium are elements with still unknown production sites and nucleosynthetic origins. Three sources have been suggested for $\mathrm{Cu}$ production: AGB stars, and stars which explode as SNe Type Ia and SNe Type II, but it is still unclear the main site of $\mathrm{Cu}$ production. For Sc the situation is even more mysterious with unclear processes and main source (Nissen et al. 2000). If the low $[\alpha / \mathrm{Fe}]$ ratios observed for the 

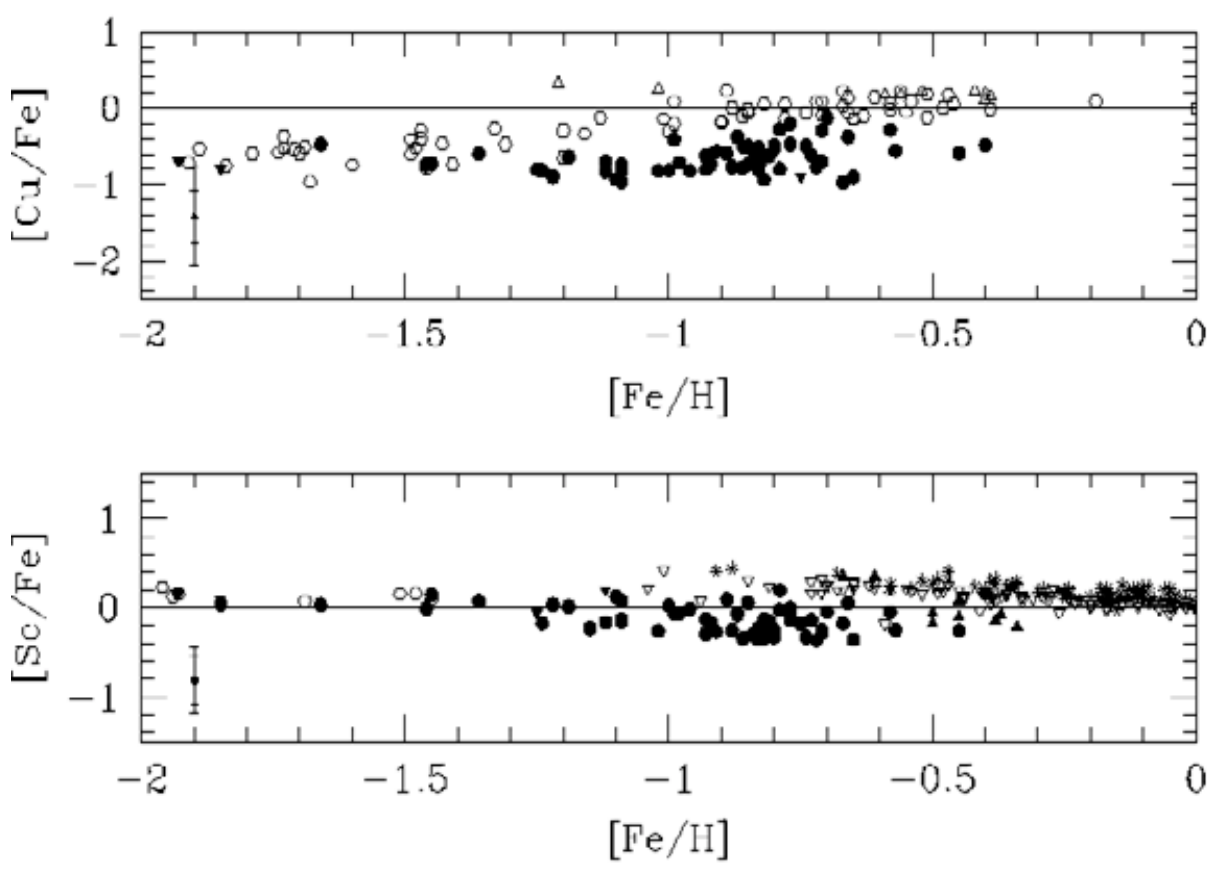

Figure 1. Copper and Scandium abundance ratios. Black dots: our sample stars. Filled triangles: stars from the LMC disk of Smith et al. (2002). Black symbols: stars from the MW: Bai et al. 2004 (open circles), Prochaska et al. 2000 (open triangles), Nissen et al. 2000 (down open triangles), Fulbright 2000 (crosses).

LMC Inner Disk stars is due to a higher content of SNe Ia relative to SNe II, the low $[\mathrm{Cu} / \mathrm{Fe}]$ behavior rules out $\mathrm{SNe}$ Ia as the main site of $\mathrm{Cu}$ production.

\subsubsection{The s-process elements}

In Figure 2 we report abundance ratios for $\mathrm{Y}, \mathrm{Zr}$ and $\mathrm{Ba}$, together with results for La given in our previous paper. As is clear from this picture, heavy and light $s$-elements behave in very different ways. Light- $s$ show low values with underabundant ratios relative to Galactic stars of the same metallicity, with a mean $[\mathrm{Zr} / \mathrm{H}]=-0.45$ and $[\mathrm{Y} / \mathrm{Fe}]=-0.30$ dex, while heavy- $s$ are supersolar, with Ba showing many enhanced values.

According to nucleosynthetic prescriptions, heavy- $s$ elements are mainly produced in metal-poor AGB stars, with a peak of production around $[\mathrm{Fe} / \mathrm{H}]=-0.7$ dex. Light- $s$ elements, on the other hand, are mostly produced in AGB stars of $[\mathrm{Fe} / \mathrm{H}] \approx-0.30$ dex. The abundances of the $s$-process elements, with a high content of heavy- $s$ and a low content of light- $s$ elements, hint for a greater contribution of metal-poor AGB stars from the generations previous to the actual. Therefore, metal-poor AGB stars had time to contaminate the ISM before star formation has been set to build such stars. Such picture is also in agreement with a slow star formation history.

\section{Abundance Relationships}

Nissen \& Schuster (1997) found two relationships between elements in galactic halo 

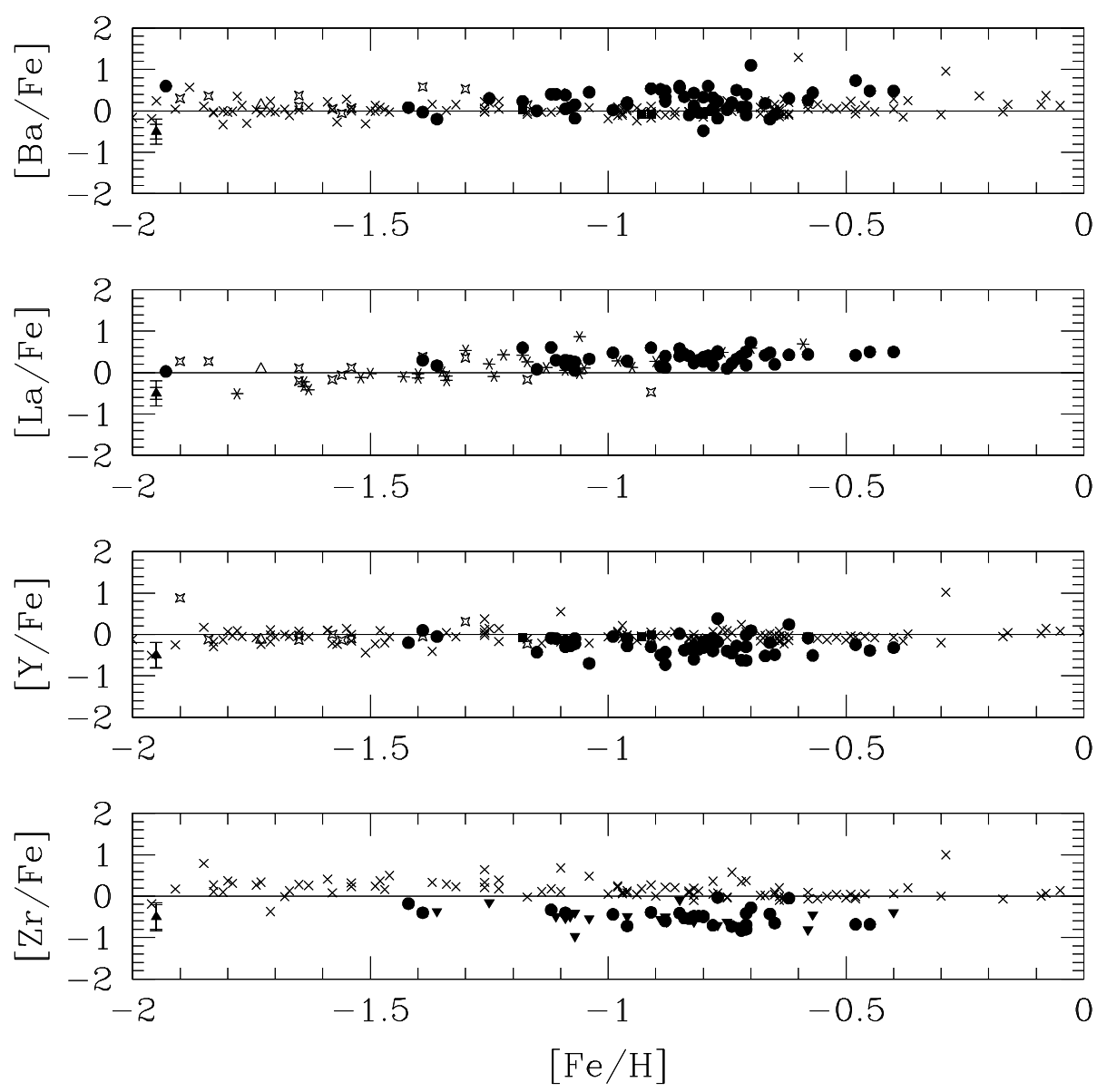

Figure 2. $s$-process elements in LMC stars. Black dots: our sample stars (the down triangles depict upper limits. Black symbols: stars from the MW: Burris et al. 2000 (stars), Fulbright 2000 (crosses).

and disk stars: they found that $\mathrm{Na}-\mathrm{Ni}$ and $\mathrm{Na}-\mathrm{Mg}$ correlate one with one the other. Such relationships were also found in some samples of stars in the dwarf spheroidal galaxies (Venn et al. 2004). In Figure 3 we have plot the $[\mathrm{Na} / \mathrm{Fe}]$ vs. $[\mathrm{Ni} / \mathrm{Fe}]$ and $[\mathrm{Na} / \mathrm{Mg}]$ vs. $[\mathrm{Mg} / \mathrm{H}]$ relationships for the LMC stars (the straight lines are the relationships derived for galactic stars (Nissen \& Schuster 1997). The upper plot of this figure shows that the Na-Ni relationship exists in the LMC stars, but maybe with a slightly different slope than that of the Galaxy. For $\mathrm{Na}$ and $\mathrm{Mg}$ we have found a clear and very different relationship compared to that derived for galactic stars. This figure strongly hints for a different SFH, leaving us with this question: how the SFH of such population developed and affected the nucleosynthesis in order to yield such a different relationship?

Finally, in Figure 4 we plot the relationship between heavy- $s$ and light- $s$ elements relative to metallicity, $[\mathrm{hs} / \mathrm{ls}]$ vs. $[\mathrm{Fe} / \mathrm{H}]$. This plot shows that the $[\mathrm{hs} / \mathrm{ls}]$ abundance ratios are high in the LMC, with a mean value of $\sim+0.65$ dex and a slow increasing trend with metallicity. From nucleosynthetic predictions (Busso et al. 1999, Busso et al. 2001 and references therein), heavy-s elements are mainly produced in metal-poor AGB 


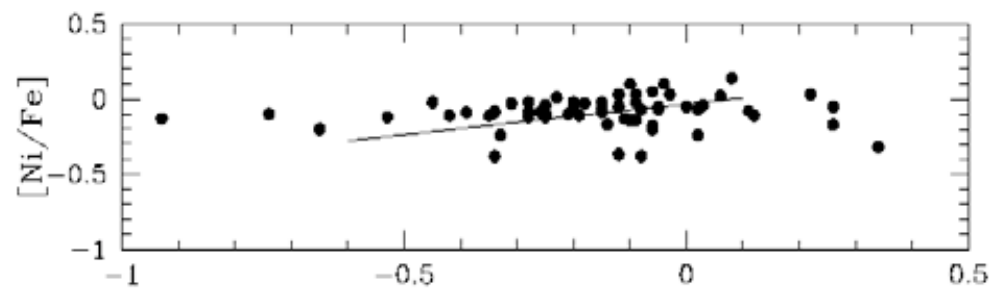

$[\mathrm{Na} / \mathrm{Fe}]$

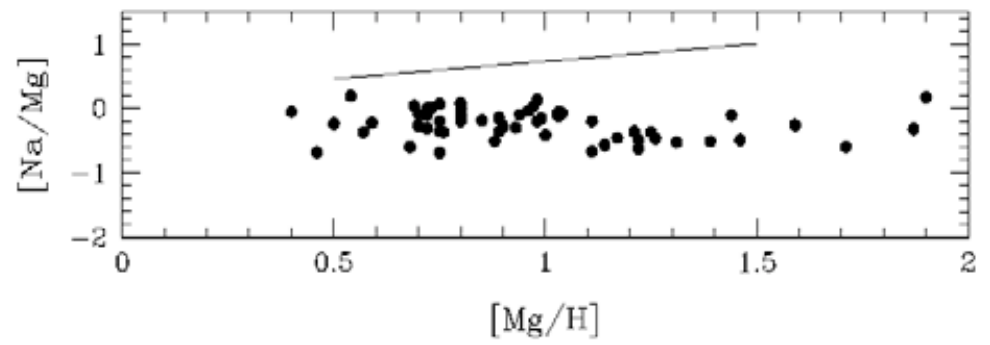

Figure 3. The Na-Ni and the Na-Mg relationships for the LMC Inner Disk sample. The straight lines are the relationships of Nissen \& Schuster (1997) for stars from the galactic halo and disk.

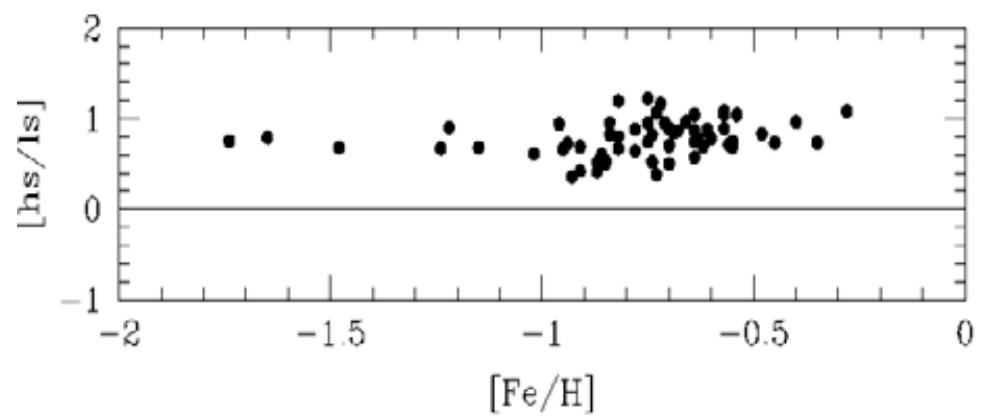

Figure 4. The heavy-s/light-s abundance ratio for the LMC Inner Disk stars.

stars, with a peak of production in $[\mathrm{Fe} / \mathrm{H}] \approx-0.70$ dex, while the light- $s$ elements are mainly produced in more metal-rich stars (with production peak at $[\mathrm{Fe} / \mathrm{H}] \approx-0.30 \mathrm{dex}$ ). This indicate that the metal-poor AGB stars had time to contaminate the ISM before the onset of a new SF process in this region. This is also in agreement with the hipothesys that such population has undergone a slow $\mathrm{SFH}$.

\section{Conclusions}

In this paper we report chemical abundances for $\mathrm{Cu}, \mathrm{Sc}, \mathrm{Y}, \mathrm{Zr}$ and $\mathrm{Ba}$, for a sample of the Inner Disk of the LMC. We have found that Sc shows a slight decrease from $[\mathrm{Fe} / \mathrm{H}]=-2$ to -1 dex, with no trend for higher metallicities. $\mathrm{Cu}$ shows a flat trend for $[\mathrm{Fe} / \mathrm{H}]<-1.0$ dex, with a mean value of $[\mathrm{Cu} / \mathrm{Fe}] \sim-0.60$, and a slow increasing trend for higher metallicities. Compared to galactic samples, $\mathrm{Cu}$ and $\mathrm{Sc}$ ratios overlap those of the galactic halo, with an offset for metallcities higher than $\sim-1.2$ dex. We have found that 
in the LMC Inner Disk stars the $\mathrm{Na}-\mathrm{Ni}$ and $\mathrm{Na}-\mathrm{Mg}$ relationships are present, although the Na-Mg relationship is very different than that derived for stars from the Galaxy. We have shown that the $[\mathrm{hs} / \mathrm{ls}]$ abundance ratios are high (mean of $[\mathrm{hs} / \mathrm{ls}] \sim-0.65 \mathrm{dex}$ ) and increases with metallicity. The high hs/ls ratios, as the low $[\alpha / \mathrm{Fe}]$ previously derived, are consistent with a slower star formation history for this population.

\section{Acknowledgements}

L.P. acknowledges CAPES and FAPESP fellowships \#0606-03-0 and \#01/14594-2 respectively.

\section{References}

Bai, G.S., Zhao, G., Chen, Y.Q., Shi, J.R., Klochkova, V.G., Panchuk, V.E., \& Qiu, H.M., 2004 A\&3 425, 671

Burris, D.L., Pilachowski, C.A., Armandroff, T.E., Sneden, C., Cowan, J.J., \& Roe, H. 2000 ApJ 544, 302

Busso, M., Gallino, R., \& Wasserburg, G.J. 1999 ARA\&A 37, 239

Busso, M., Gallino, R., Lambert, D.L., Travaglio, C., \& Smith, V.V. 2001 ApJ 557, 802

Fulbright, J.P. 2000 AJ 1201841

Gilmore, G. \& Wyse, R. 2004 astro-ph/0411714

Kurucz, R.L., Furenlid, I., \& Brault, J. 1984 National Solar Observatory Atlas, Sunspot, New Mexico: National Solar Observatory 1, 984

McWilliam, A. \& Smecker-Hane, T.A. 2004 AJ astro-ph/0409083

McWilliam, A. 1998 AJ 115, 1640

Nissen, P.E. \& Schuster, W.J. 1997 A\&A 326, 752

Nissen, P.E., Chen, Y.Q., Schuster, W.J., \& Zhao, G. 1997 A $\& A$ 353, 722

Pompéia, L., Hill, V., \& Spite, M. 2004 Proc. of the VIII Symposium Nuclei in the Cosmos in press

Prochaska, J.X., Naumov, S.O., Carney, B.W., McWilliam, A., \& Wolfe, A.M. 2000 AJ 120 2513

Shetrone, M.D., Venn, K.A., Tolstoy, E., Primas, F., Hill, V., \& Kaufer, A. 2003 AJ 125, 684

Smecker-Hane T., McWilliam, A. 2002 astro-ph/0205411

Smith, V.V., Hinkle, K.H., Cunha, K., Plez, B., Lambert, D.L., Pilachowski, C.A., Barbuy, B., Melndez, J., Balachandran, S., Bessell, M.S., Geisler, D.P., Hesser, J.E., \& Winge, C. 2002 astro-ph/0205411

Tolstoy, E., Venn, K.A., Shetrone, M.D., Primas, F., Hill, V., Kaufer, A., \& Szeifert, T. 2003 AJ 125, 707

Venn, K.A., Irwin, M., Shetrone, M.D., Tout, C.A., Hill, V., \& Tolstoy, E. 2004 AJ 128, 1177 